\title{
Tensile Testing of Cellulose Based Natural Fibers for Structural Composite Applications
}

\author{
MARK C. Symington, W. M. BANKS* AND Opukuro DAvid WeSt \\ Department of Mechanical Engineering \\ University of Strathclyde, Glasgow, G1 1XJ, UK \\ R. A. PETHRick \\ Department of Pure and Applied Chemistry \\ University of Strathclyde, Glasgow, G1 IXJ, UK
}

\begin{abstract}
A series of tensile tests were conducted on a Lloyd LRX tensile testing machine for numerous natural fibers deemed potential candidates for development in composite applications. The tensile tests were conducted on the fibers jute, kenaf, flax, abaca, sisal, hemp, and coir for samples exposed to moisture conditions of (1) room temperature and humidity, (2) $65 \%$ moisture content, (3) $90 \%$ moisture content, and (4) soaked fiber. These seven fibers were then tested for the four conditions and the mechanical properties of tensile strength, tensile strain to failure, and Young's modulus were calculated for the results. These results were then compared and verified with those from the literature, with some of the fibers showing distinctly promising potential. Additionally, a study on the effect of alkalization using $3 \% \mathrm{NaOH}$ solution was carried out on flax, kenaf, abaca, and sisal to observe impact that this common fiber pre-treatment process has on fiber mechanical properties. The result of the investigation indicated that over treatment of natural fibers using $\mathrm{NaOH}$ could have a negative effect on the base fiber properties. It is consequently apparent that a treatment time of less than $10 \mathrm{~min}$ is sufficient to remove hemicelluloses and to give the optimum effect.
\end{abstract}

KEY WORDS: natural fiber, jute, kenaf, flax, sisal, coir, abaca, alkalization, moisture, tensile testing.

\section{INTRODUCTION}

$\mathbf{P}$ REVIOUS RESEARCH SUGGESTS that fibers such as flax or hemp exhibit only moderate mechanical properties when processed into composites such as those in Figure 1. This prevents their use in high-performance applications (e.g., where carbon reinforced composites would be utilized), although it is recognized they can compete with glass fibers

*Author to whom correspondence should be addressed. E-mail: bill.banks@strath.ac.uk

Figures 1-17 appear in color online: http://jcm.sagepub.com

Journal of COMPOSITE MATERiAls, Vol. 00, No. 00/2008 


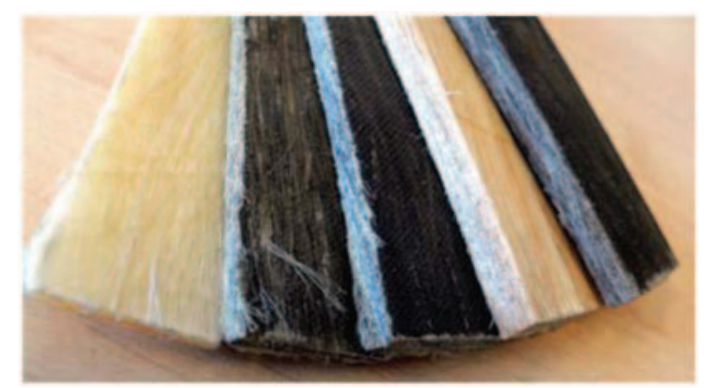

Figure 1. From left to right, sisal, flax, hemp, abaca, and kenaf natural fiber composites.

for many reasons. However this is not the end of the road for natural fibers. It is suggested that the potential of using natural fibers in composite applications has not been fully realized, as the full strength of fiber building blocks or 'microfibrils' is not being utilized in the raw materials. Potential 'infusion' of resin into suitable natural fiber mini superstructures is speculated to release much more of the potential that natural fibers have to offer. Adding sol gel and exfoliated clay to this material produces a nano-material with improved toughness and fire resistance. It also permits the natural fiber composite to be used in multi-functional structural applications. Further to this, the production of these advanced natural fiber based composites would have a variety of desirable properties, especially in the present climate of drive towards sustainability.

Most cellulose-based fibers are a renewable resource, and the production requires little energy, $\mathrm{CO}_{2}$ is used while oxygen is given back to the environment. Additionally thermal recycling is possible, where glass and man made fibers often cause problems in combustion furnaces.

Therefore, cellulose based composites would offer the benefits of recycling and under optimal use of resources, relatively low cost composites.

At present there are a number of mechanical properties available for cellulose based natural fibers in the literature. Many of the cited values for tensile strength $(\sigma)$, breaking strain of the fiber $(\varepsilon)$ and also Young's modulus $(E)$ vary dramatically from scattered sources. Due to this, there is also likelihood that testing conditions are non-standard for obtaining the mechanical properties of the natural fibers.

A more comprehensive collection of the mechanical properties from tensile testing of natural fibers will therefore be extremely useful in aiding the further development of natural fiber based composites. The aim of the present study is to provide this, and additionally confirm that the mechanical properties of the sourced project fibers concur with the various literature values exhibited. It is also of interest to investigate the resultant effect of alkalization on the fiber mechanical properties, a common fiber pre-treatment used to increase fiber/matrix interfacial strength.

\section{FIBRES}

\section{Introduction to Fibers}

From a literature research, a number of commercially available natural fibers have been identified for use, and are shown in Figure 2. Some of these fibers present excellent specific mechanical strengths, comparable to, or exceeding many composite fibers used in industry. 

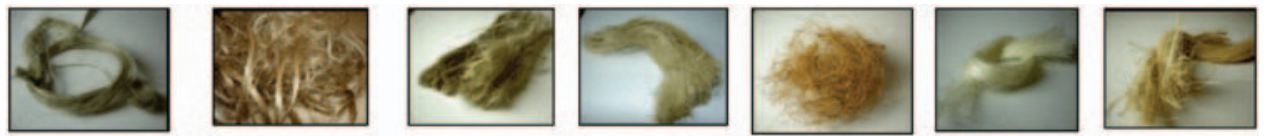

Figure 2. From left to right, flax, jute, kenaf, hemp, coir, sisal, and abaca.

The main fibers targeted and sourced for use in the research are the bast fibers jute, kenaf, hemp, and flax, the leaf fibers abaca and sisal and also the fruit husk fiber coir. Each of these fibers have varying amounts of the main building blocks, and of the fiber structure, cellulose, along with other influential constituents.

From the tensile testing of the fibers not only the mechanical properties are sought. It is of great benefit to have an insight into failure mechanism of the fibers. This will help to identify fiber suitability for use in target composite applications.

\section{Fiber Cross-sectional Areas}

The target fibers in this study are in general non-uniform through their cross-section. This presents a number of problems for tensile testing, especially when combined with variation of cross-sectional shape along the fiber length. Additionally, as failure may always occur at any section of weakness along the fiber, it is difficult to predict the break point. This makes it difficult to obtain an exact measure of cross-section. Again, this problem is also compounded by the splintering failure methods observed in the fibers due to their microfibril structure resulting in cross-section variation throughout testing.

From the complexities of obtaining cross-sectional areas of natural fibers, it could be said that there are difficulties in obtaining exact values of tensile strength $(\sigma)$ and therefore Young's modulus $(E)$ for the fibers. Rao et al. [1] highlighted similar issues, and investigated natural fiber cross-sections with optical laser equipment. It was found that fiber diameter varied greatly, however much of the fiber may have been applicable to estimate cross-section as oval or circular. The tensile testing was then carried out using relative shapes of cross-section rather than actual cross-sectional area. This approximation was carried out using a digital micrometer. This method was deemed acceptable in their studies to give approximate results.

In this study, it is also a requirement for approximate results, therefore a similar method is used for measuring cross-sectional area. This was deemed appropriate as the requirements for testing results are to (1) confirm the quality of natural fiber obtained for the research work, and (2) add a comprehensive set of approximate tensile testing results from the literature. During the tensile testing, Rao et al. indicated taking an average of five tensile test results for each fiber in their testing. For this study it was deemed that due to the potential variability in natural fiber properties, 25 tests per fiber might help to increase the quality of approximation. This was felt necessary due to the errors already associated with measuring the cross-sectional areas.

In Table 1, the photomicrographs of fiber cross-section for jute, hemp, flax, kenaf abaca, sisal, and coir can be viewed. The natural fibers were infused in resin, cut through the cross-section, and surface polished to allow for inspection. The cellular structure of the fibers is clearly evident, along with the variability in fiber size and shape. For non-circular fibers, intuitive approximation had to be carried out by carefully rolling the fiber into an approximate circular cross-section and measuring the diameter. The fibers are then straightened out before mounting in the fiber grips. 
Table 1. Photomicrographs of fiber cross-sections at $\times 50$ and $\times 200$ respectively.

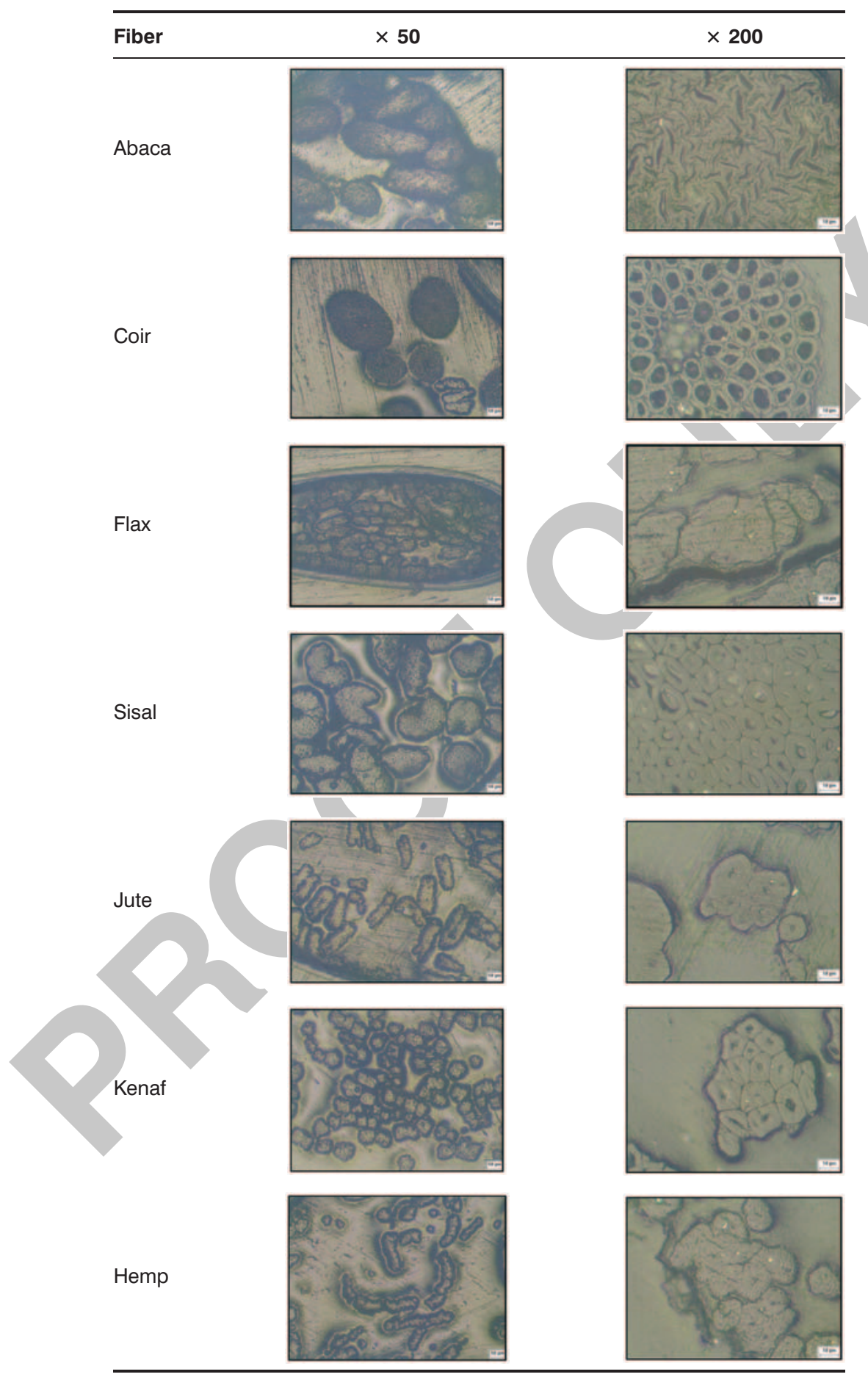




\section{TESTING PROCESS}

\section{ASTM Standard}

For the tensile testing of natural fiber, the closest applicable standard used was ASTM D 3822-01 the 'Standard for Tensile Properties of Single Fibers'. This ASTM standard is typically used to quantify the mechanical properties of textile fibers and threads, which are often from a natural source, such as flax or cotton. This provides a good guideline as it is on the correct testing scale with the fibers being fine in cross-section. Table 2 highlights the key testing areas derived from the ASTM standard and discusses their importance in the testing procedure. Information on the testing equipment used is given in the following section.

\section{Testing Equipment}

As well as using the ASTM standard for the testing procedures for the fibers themselves, the ASTM standard for the specification of testing equipment also has to be met. This was found to be ASTM D 76-99 the 'Standard Specification for Tensile Testing Machines for Textiles'. The Lloyd LRX tensile testing machine shown in Figure 3 meets these requirements.

Again, to ensure best practice, a general testing methodology was established for one individual fiber test. Testing was then carried out 25 times for the seven target fibers, flax, jute, kenaf, sisal, hemp, abaca, and coir to aid in accuracy of results and to indicate variations from quoted values.

Table 2. The key testing points that have been summarised from the ASTM D 3822-01 Standard.

Key testing
area \begin{tabular}{l} 
Description \\
\hline $\begin{array}{l}\text { Constant rate } \\
\text { of extension }\end{array}$ \\
$\begin{array}{l}\text { The rate of extension of pull of the tensile testing machine has to be accurate and } \\
\text { consistent for all the testing batches. If this is altered for each batch, discrepancies } \\
\text { may appear in results. In the testing for this study the rate of extension or pull was } \\
\text { set } 10 \mathrm{~mm} / \mathrm{min} \text { for all the fiber batches. } \\
\text { The ASTM standard highlights the importance in preventing the slippage of the } \\
\text { testing fiber through the fiber grips and therefore has to be avoided. Fiber slippage } \\
\text { grips slippage in the true levels of fiber extension at maximum load and negatively } \\
\text { influences the accuracy of the failure strain and Young's modulus significantly. } \\
\text { Fiber slippage was carefully watched for during testing. } \\
\text { The alignment of the fibers in the jaws of the fiber grips has to be straight and square. } \\
\text { This is to ensure that during testing the fiber is not being pulled at an angle to give } \\
\text { distorted mechanical properties. } \\
\text { The gauge length or the 'pull length' is the distance between the edge of the upper } \\
\text { and lower fiber grips. This is the actual length of fiber being pulled during testing } \\
\text { and it is important this is kept consistent throughout testing. The gauge length for } \\
\text { testing was kept at } 70 \text { mm. } \\
\text { When aligning the fiber in the tensile testing machine grips, the twirling or twisting of } \\
\text { fibers before being gripped has to be removed to ensure any torsional twisting } \\
\text { during pull is removed. This twisting, again, may prematurely promote or suppress } \\
\text { failure of the fiber, so therefore should be avoided to obtain true values. }\end{array}$ \\
Gauge length
\end{tabular}




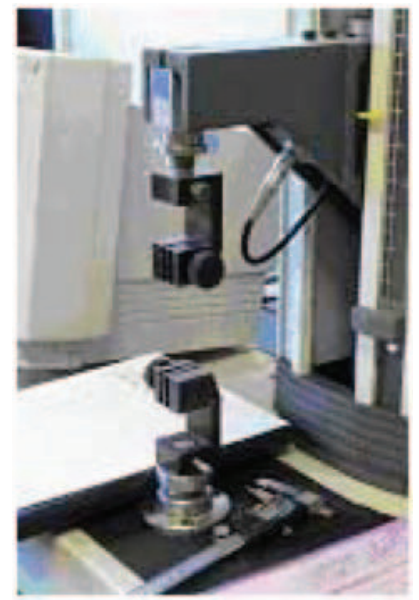

Figure 3. The Lloyd LRX tensile testing machine used for the tensile testing of the natural fibers. It is connected to a control computer from which graphical results can be obtained.

\section{MOISTURE STUDIES}

\section{Aims}

Along side testing the base fiber at room temperature/humidity it was also considered beneficial to conduct moisture studies on the natural fibers. This would aid in quantifying the effect moisture exposure has on the mechanical properties of the natural fibers. The moisture exposure studies on the fibers were carried out using dessicators.

\section{Dessicators}

There are seven target fibers under investigation, and to conduct research into the influence of how moisture can affect the mechanical properties of each of these fibers, exposure of the fibers to different moisture levels is necessary. Four moisture exposure levels for each fiber are thought to be a suitable number to gather any trends or influences that moisture has on the fiber mechanical properties. These moisture levels are: (1) standard room humidity, otherwise the standard unprocessed fiber tested straight in the machine; (2) $65 \%$ moisture exposure; (3) approximately $90 \%$ moisture exposure, and (4) soaked fibers.

Fiber moisture level (1) was just the standard fiber at normal room temperature and humidity, level (4) was the fiber samples completely immersed in jars of water for a set period of time at a controlled temperature to ensure water saturation. Notes on the soaked fiber (4) are found in the next subsection. The moisture exposure levels of $65 \%$ and $90 \%$ were created in a controlled environment for the required time. This was conducted using a dessicator as shown in Figure 4, by placing it in a sealed temperature controlled oven with the appropriate dessicator solution in the base to control the humidity. For $65 \%$ and $90 \%$ humidity exposures, the fibers were in the dessicator and temperature controlled oven for 6 weeks. This length of time was perceived to be adequate from previous studies to allow moisture levels of the fibers to equilibrate to the controlled environment. When fiber testing was undertaken, specimens were removed from the dessicator in small quantities 


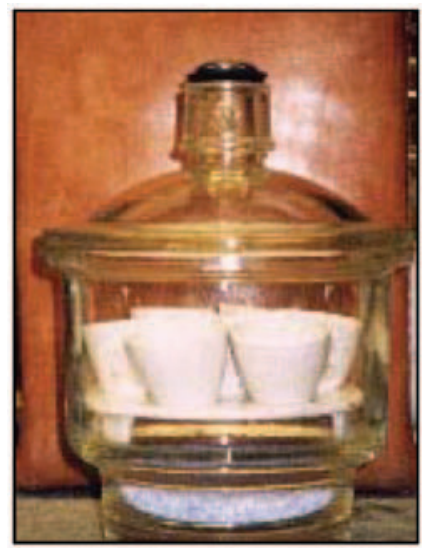

Figure 4. A dessicator similar to that used for controlling humidity.

and tested swiftly to reduce the possibility of moisture change due to differences in environment. This was also the case for the soaked fibers in the tensile testing, however the excess water was removed from these. More information on the soaked fibers can be found in the following section.

\section{Soaked Fiber}

Batches of the seven target fibers, flax, jute, kenaf, sisal, hemp, abaca, and coir, were soaked in water in sealed individual jars for a period of a week in a temperature controlled environment. The weights of the fibers before and after soaking were measured in order to calculate the percentage volume and percentage weight of water contained within each fiber. The weights noted before and after soaking can be viewed in Table 3; the densities taken from the literature used in the calculations are also included. The values measured were then used to calculate the percentage moisture content by weight using Equation (1) below, where $W_{0}$ is the initial measured fiber weight and $W_{t}$ is the final weight of the sample fiber after soaking for one week.

$$
M_{t}=\frac{W_{t}-W_{0}}{W_{0}} \times 100
$$

The moisture content as a percentage of weight of the initial fiber can be viewed in Figure 5. These values were then used to calculate the moisture content as a percentage of volume using the relationship density, $\rho=$ mass/volume. As we have the mass of water soaked in the fiber and the density of water is $1000 \mathrm{~kg} / \mathrm{m}^{3}$, the volume of water as a percentage of initial fiber volume is relatively straightforward to calculate. These results are also shown in Figure 5. From the results of the calculations, the ascending orders of moisture content as a percentage weight are shown in Table 4. It can be seen that the fruit fiber coir $(70.49 \%)$, from coconut husks has the least capacity to hold water in its fiber structure, this may be partly explained by the fiber structure, where there are many 'air chambers' to make the coir fiber 'springy'. The purpose of the springy fibers on the husk is 
Table 3. The measured weight of fibers before and after soaking. Used to calculate water uptake.

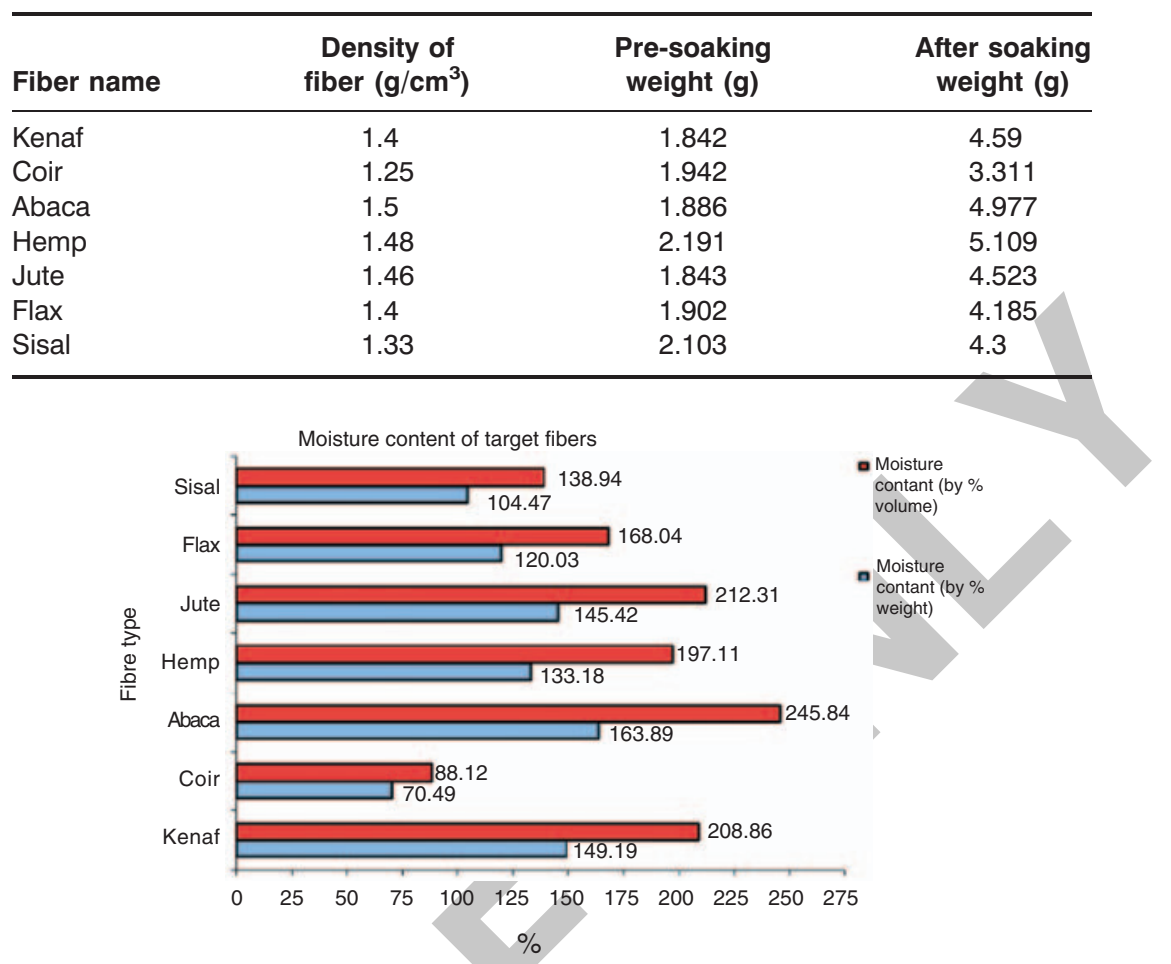

Figure 5. Percentage moisture content by weight and by volume for the soaked fibers.

Table 4. Ascending ordering of percentage weight of water uptake of soaked fibers.

\begin{tabular}{lcc}
\hline $\begin{array}{l}\text { Fiber } \\
\text { name/rank }\end{array}$ & Fiber type & $\begin{array}{c}\text { Moisture content } \\
\text { (\% weight) }\end{array}$ \\
\hline (1) Coir & Fruit fiber & $70.49 \%$ \\
(2) Sisal & Leaf fiber & $104.5 \%$ \\
(3) Flax & Bast fiber & $120.3 \%$ \\
(4) Hemp & Bast fiber & $133.18 \%$ \\
(5) Jute & Bast fiber & $145.42 \%$ \\
(6) Kenaf & Bast fiber & $149.19 \%$ \\
(7) Abaca & Leaf fiber & $163.89 \%$ \\
\hline
\end{tabular}

to protect the coconut from damage when falling from the tree by reducing the impulse during impact. These 'air chambers' may not allow water to penetrate and partly explains why the fiber does not hold as much water as the other fibers.

The two leaf fibers sisal (104.5\%) and abaca (163.89\%) show a significant variability in moisture content, which indicates that although from leaves of plants, their function within the leaf may be somewhat different. With abaca having an extremely high ability to hold water, this may be due to the fiber's purpose in the leaf of circulating water around the plant. 
It may also reflect upon the water availability in the growing environment of the plants country where the fiber has been optimised for circulating certain fluid volumes and hence the difference in ability to hold moisture between sisal and abaca.

It is interesting to see that all the bast fibers, are falling in a similar range of ability to hold water from the calculation results. This again is possibly down to the fiber's base function when in the plant that determines the fiber structures. Bast fibers are the strong fibers in the phloem of a number of dicotyledons (a group of flowering plants). They conduct synthesized nutrients from the leaves to other parts of the plant, for example in vascular plants the phloem is the living tissue that carries organic nutrients such as sucrose to parts of the plant feeding off the stems circulation. The bast fibers in the phloem also provide structural integrity to the plant. The mixed purpose of providing strength and nutrient circulation (often in fluid form) indicates that bast fibers are designed to provided strength under moist conditions. It is interesting to see that both Jute (145.42\%) and kenaf $(149.19 \%)$, which are very similar in plant fiber origin, have a very similar ability to hold water. This is reassuring to observe as it helps in gaining confidence in measured results as these fibers have a similar plant structure.

The plant structures of the fruit, bast, and leaf fibers all have interesting characteristics that are suspected of having an influence on their ability to hold moisture. It is therefore thought that the plant fiber structures will also influence the particular fiber's mechanical behavior under various circumstances. From research it is strongly suspected that a deep understanding of the target plant fibers structure and behavior under various environmental loading conditions will help to fully release the potential strength of the fibers for given applications.

\section{TEST RESULTS}

\section{Room Temperature/Humidity}

From conducting the tensile testing of the natural fibers, graphs that offer a typical representation of the mean properties for flax, jute, kenaf, sisal, hemp, abaca, and coir can be viewed in Figure 6.

These fibers were tested at room temperature and humidity exposure. From the graph, flax displays the largest Young's modulus of $66 \mathrm{GPa}$ with a standard deviation of $( \pm 22.2)$, whilst generally still maintaining fiber integrity. The other bast fibers jute, hemp, and kenaf display results of $28 \mathrm{GPa}( \pm 8.3), 64 \mathrm{GPa}( \pm 55.5)$, and $34.2 \mathrm{GPa}( \pm 17.2)$, respectively. Testing of the leaf fibers gave Young's modulus to be $23.4 \mathrm{GPa}( \pm 6.4)$ and $17.4 \mathrm{GPa}( \pm 8.4)$ for abaca and sisal, respectively. The fruit husk fiber coir had a much lower average Young's modulus of $2.66 \mathrm{GPa}( \pm 1.76)$ compared to the other tested fibers. The test results in Figure 6 fall reasonably into the range of values exhibited in the literature for each. This provides reassurance that the fibers obtained for the research are suitable for developing into cellulose-based nanocomposites.

\section{Published Literature Values}

It is extremely important for this work that our tensile testing results for the seven target fibers are verified with those obtained by others in the academic community. 


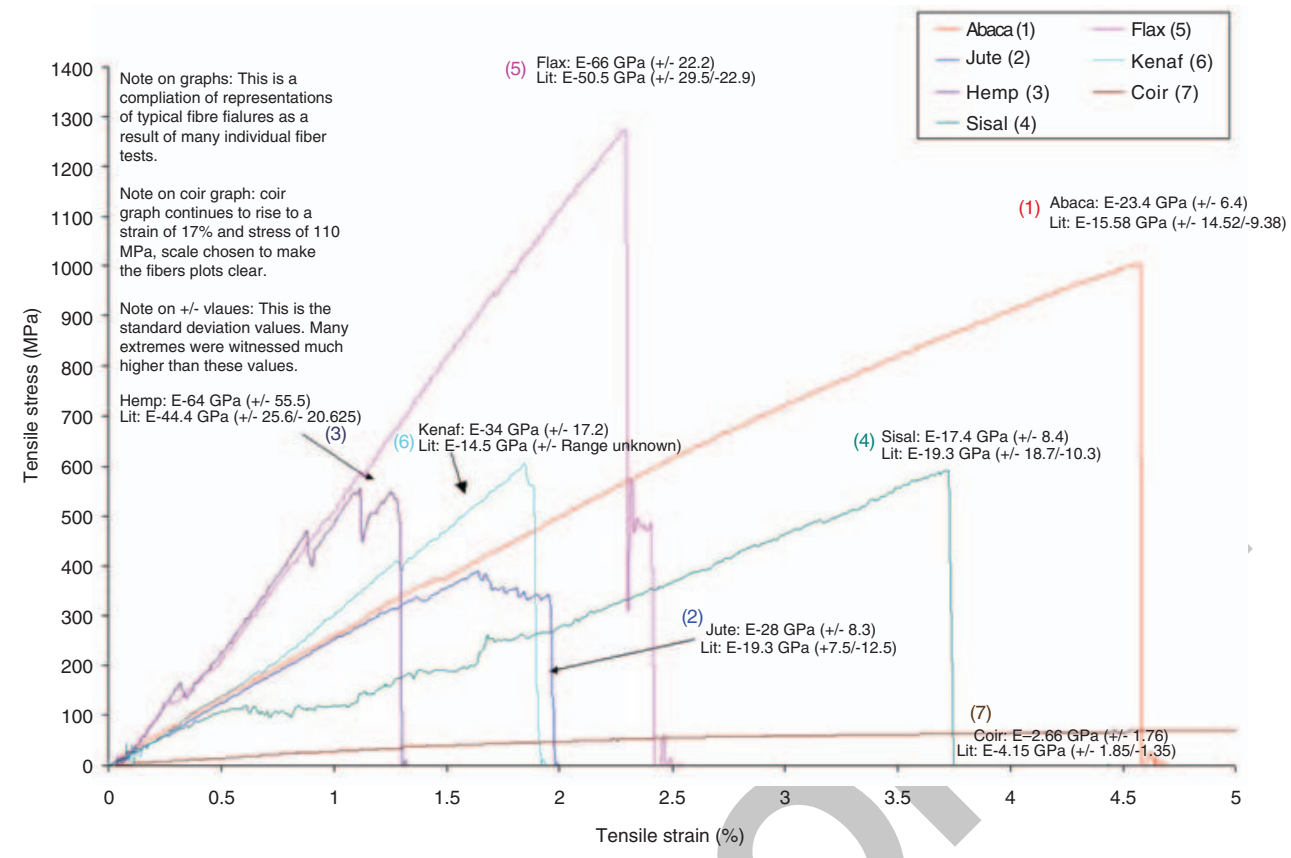

Figure 6. Tensile stress vs. tensile strain for typical tested natural fiber from sample batch.

Many published values have been gathered and can be observed in Table 5. One of the first observations made in this table is the large variability in published values, and in fact some of which were extremely debatable in terms of validity and therefore were left out. This may be due to the large variability natural fiber quality as well as sub species of fibers being tested under a general umbrella of the fiber name. From the testing conducted in this research, the standard deviation was very high for many of the fibers from the same batch which again highlights variability in tensile properties of natural fibers.

The values included in Table 5 are used in the graphical results in the following sections in the bar graphs rounding up the findings of both the raw fiber and moisture-exposed fiber. Comparisons to our tested values for mechanical properties will be made with those from literature with additional comments and discussions.

\section{Influence of Moisture on Tensile Strength}

The results for tensile strength $(\sigma)$ for the moisture studies are given in Figure 7. It is interesting to see that there is a range of effects that moisture has on the tensile strength of the target fibers. Generally from observing the graph, it can be said that moisture plays a significant role in influencing the mechanical properties of most of the fibers. Whilst fibers such as kenaf, jute, and abaca centre around similar values when fully soaked to that of initial room temperature/humidity conditions, fibers such as flax and kenaf take a notable decrease in tensile strength. With the likes of hemp proving unable to be tested, questions are raised regarding its stability when exposed to various environmental conditions.

The returned values of tensile strength for all the testing conditions have all been collated and compared to the available literature values, also in Figure 7. From this graph, 
Table 5. Gathered mechanical properties for target fibers from published literature.

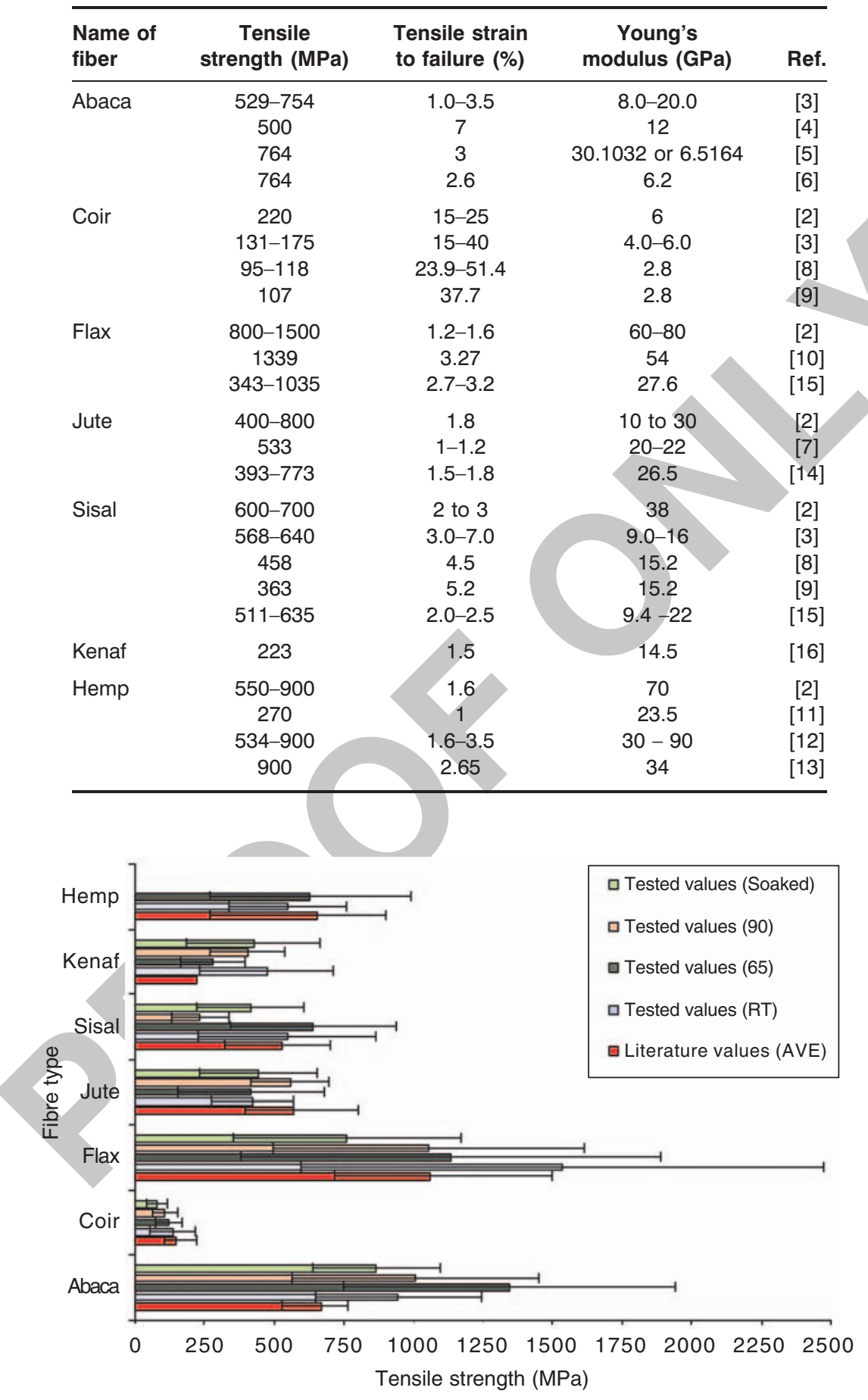

Figure 7. Comparison between tested results and literature values for tensile strength. 
it is reassuring to see that most of the values returned from tensile strength during the mechanical testing of the sourced target fibers are falling in the range of the literature values. From the tests that could be conducted for hemp, the values returned also fall in the literature value range with the standard deviation for our tests being very similar. Only one value for the kenaf fiber could be found from the articles readily available, due to this no data range could be conveyed. This value seems to be slightly low in comparison to our tested values, but is in the approximate region. For the sisal fiber the results are again similar to literature, however the dip at $90 \%$ humidity exposure is as yet unexplained and it may be necessary to investigate what causes this effect further on in the research work.

The abaca and flax fiber samples used in the testing appear to be of good quality from the results. The results are overlapping into the literature value range for both these fibers, although the standard deviation for the tested abaca and flax are considerably above the quote literature values. This implies that the samples of each of these fibers are of very good quality, or perhaps testing error is warping results even though endeavors have been made to reduce potential error sources. The values for coir and jute also seem to conform to the literature values which are also very reassuring that testing procedure may not be influencing accuracy.

\section{Influence of Moisture on Tensile Strain to Failure}

The results for the tensile strain at break for the natural fibers are shown in Figure 8. From testing, the fibers return tensile failure strains that concur with literature values with reassuring accuracy, the reasons for fiber trends over the humidity ranges are harder to identify.

Kenaf and jute are seen to only vary $25 \%$ over the humidity range with a slight dip in the value of strain for the soaked fiber. This may be due to swelling effects induced in the fiber causing radial tension. The consequence of this may be an influence on the tensile failure strain that can be withstood. This noticeable dip may also be witnessed for the soaked flax

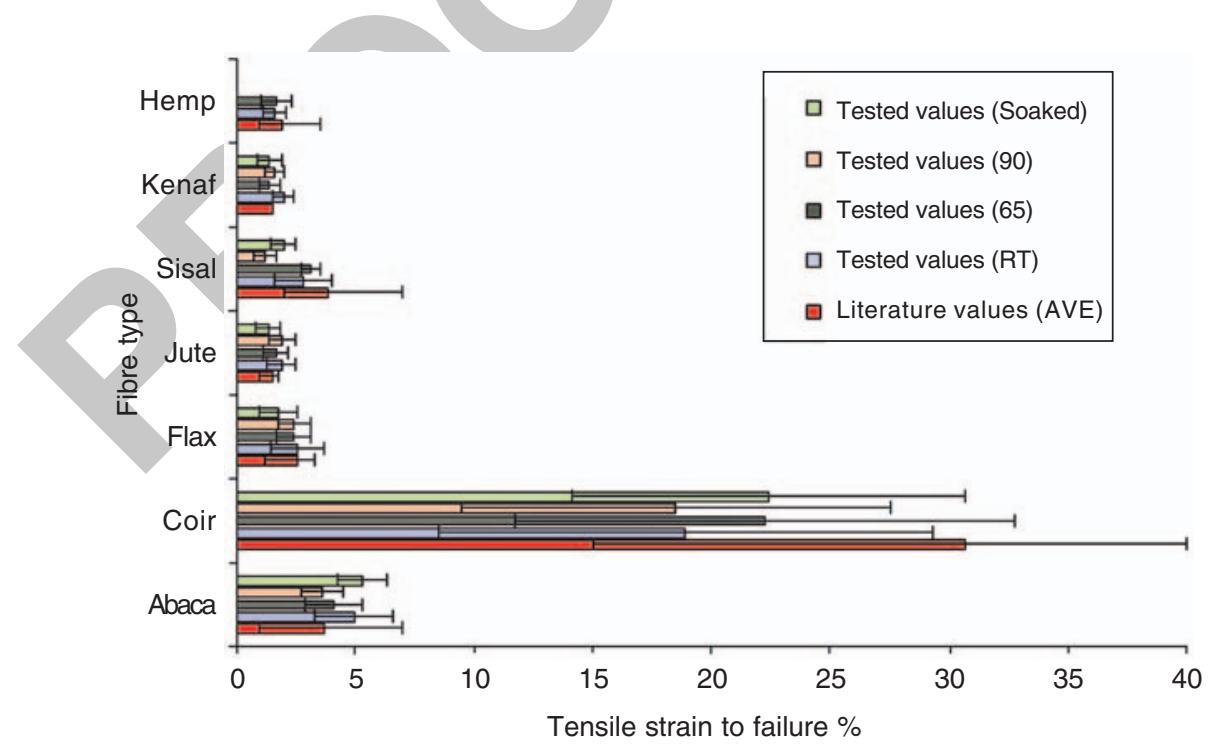

Figure 8. Comparison between tested results and literature values for tensile strain to failure. 
fiber, a bast fiber like jute and kenaf. Conclusive comments cannot be made for hemp as this was unable to be tested fully due to fiber breakdown from moisture exposure.

With regard to the leaf fiber sisal it seems the pattern of behavior is harder to understand with quite a range in tensile failure strain. The spread of the initial failure strains for the room temperature/humidity batch has a considerable standard deviation, and from $65 \%$ onwards the standard deviation for the humidity in the batches narrows considerably to a constant level indicating consistency in testing. What is apparent from the testing of sisal is that moisture exposure has a significant effect on the fiber's tensile strength and strain.

For abaca, the average failure strain fluctuates by around $20 \%$ over the humidity testing range with an upturn in the strain value for the soaked batch. The exposure of abaca to moisture is suspected to relax the fiber structure slightly as the hemi-cellulose sub-matrix in the fiber is accommodating to the presence of the water molecules and provides more relaxation in the fiber structure. It is also the case that the failure stress decreases with increasing moisture exposure. This may also be attributed to the vulnerability of cellulose when exposed to moisture [2].

\section{Influence of Moisture on Young's Modulus}

From observing the set of results for Young's modulus, a few interesting points can be made. From observing Figure 9, both kenaf and jute behave in a similar manner, taking an upturn in the tested values for Young's modulus as the humidity exposure increases, and then after a certain point, a distinct decrease. This perhaps suggests that the fibers swell, with the radial tension increasing the fibers' Young's modulus. However, after a certain degree of swelling the fibers are vulnerable and hence a lowering in mechanical properties occurs $[2,10]$.

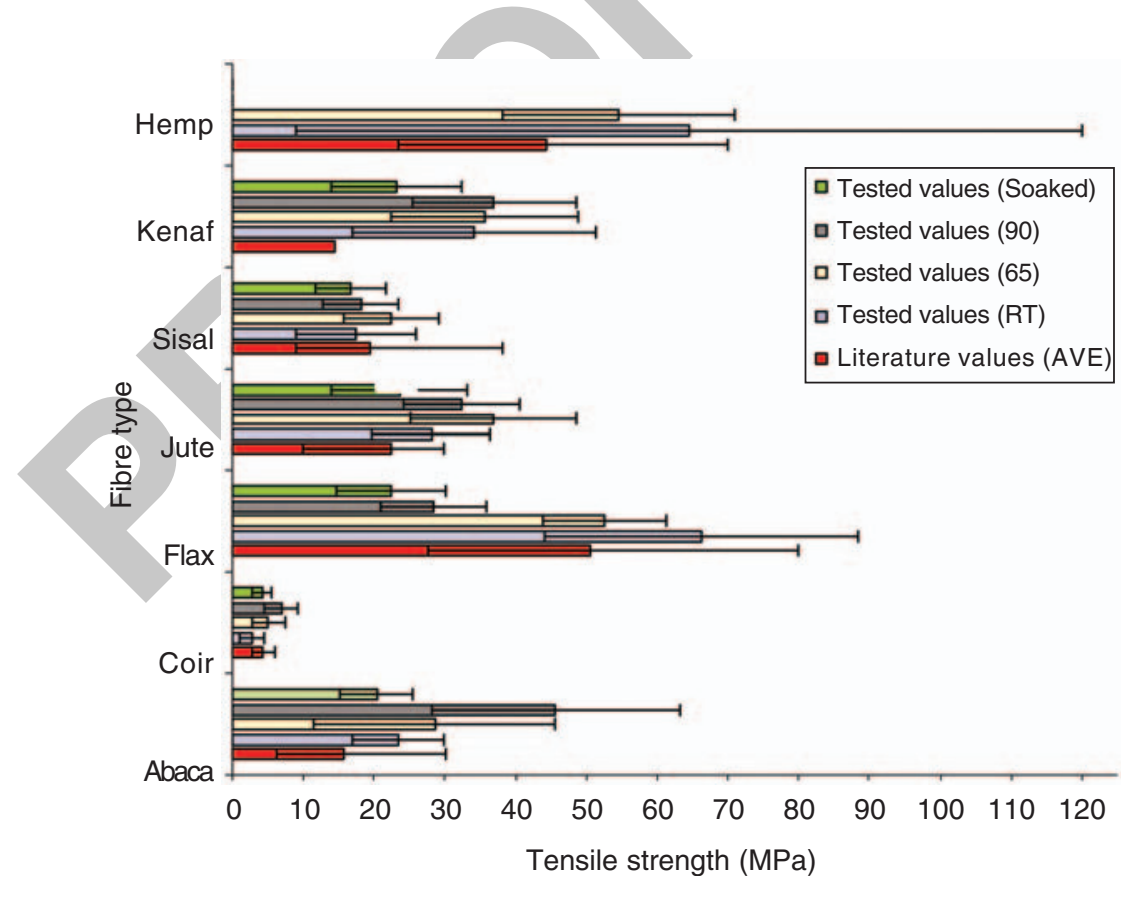

Figure 9. Comparison between tested results and literature values for Young's Modulus. 
As mentioned previously, jute and kenaf are from a similar family of fibers (and are therefore similar in structure). Therefore, to see the same patterns in results for these fibers is reassuring towards validity. With regard to the pattern exhibited by the flax fiber for Young's modulus, there is a significant decrease with increasing moisture content. Flax is observed to have the highest cellulose content of all the fibers, therefore the degree of swelling may be greater. This could suggest that the degree of vulnerability when exposed to moisture is also greater. It is also observed for flax and most of the fibers, that as the moisture exposure increases, the standard deviation about the average reduces. This indicates more predictable failure envelope, which may be due to relaxing the fiber structures and stress concentrations in the mini super structures [2].

For the leaf fiber sisal it is very surprising to see a reasonably consistent value of Young's modulus in comparison to other fibers. This is considering the varying pattern observed in the stress graph (Figure 7) and strain graph (Figure 8) for the fiber. This suggests there are some unusual patterns of behavior of the fiber structure, and there perhaps may be some internal fiber characteristics to maintain stiffness when exposed to varying levels of moisture. It may also be that the sisal fiber is required to remain at consistent stiffness for varying levels of circulation of water in the leaf, which makes intuitive sense. The other leaf fiber, abaca, increases in Young's modulus as moisture exposure is increased, and dips considerably once soaked. It is also apparent that abaca still maintains considerable strength throughout the moisture studies.

Further to the investigation into the base mechanical properties of the natural fibers and the effect of moisture it is also of use to investigate the effect of alkalization on the mechanical properties of certain fibers.

\section{ALKALIZATION OF NATURAL FIBRES}

\section{Alkalization Literature Background}

Alkalization is a common pre-processing technique used on base natural fiber to remove hemicelluloses, fats and waxes that may reduce the interfacial strength when processed into composite form. It is of great interest to understand the effect this has on the base fiber mechanical properties, as whilst it may ultimately increase the resultant composite strength through increasing fiber matrix adhesion, the strength of the fiber itself may reduce. Due to this, to allow fuller understanding of the process effects on mechanical properties it is thought tensile testing of alkalised fiber under the same conditions considered previously is extremely beneficial. It is additionally beneficial to investigate current literature to aid in understanding of other effects that alkalization may have. Some of these can be found in Table 6 .

Alkalization aims to remove hemicelluloses from natural fiber, which often results in a change in fiber surface energy in a polar or dispersive manner [2]. Hemicellulose, which is thought to consist principally of xylan, polyuronide and hexosan has been shown to be very sensitive to caustic soda. The caustic soda (sodium hydroxide) is said to exert only minimal influence on the lignin in the fibers and the high strength alpha-cellulose. Other effects reported from alkalization of natural fibers are shown in Table 6.

From the information in Table 6 , an indication of the effect that alkalization has on the fibers properties can be obtained. However, it is also essential to know to what effect concentration and process time has on the resulting mechanical properties of the fibers. 
Table 6. Alkalization effects and observed behavior of treatment from literature.

Observation
(1) Removal of hemicelluloses
cause the interfibrillar regions of
the fiber to becomes less dense
and rigid.
(2) Removal of hemicelluloses
in the interfibrillar regions also
softens the interfibril connections.
(3) Partial removal of Lignin
during Alkalization of the fibers

(4) Change in crystallinity through alkaline treatment reported in coir and flax.

(5) Treatment with $\mathrm{NaOH}$ leads to a decrease in the spiral angle.

(6) Randomness introduced in the orientations of the crystallites due to the removal of non-cellulosic matter.

(7) More imperfections and crevices noticed on fiber surface

(8) Lower ability of treated fiber to absorb moisture

(9) Change in proportion of alpha cellulose and cellulose-II.

(10) Swelling of the fiber during treatment

(11) Breakage of the fiber during alkalization.

(12) Uncontrollable swelling of the resulting composite after alkalization.

\section{Comment}

Fibrils are now more capable of rearranging themselves along the direction of tensile deformation. This promotes even load distribution in the fibers and reduces stress concentration likelihood.

Adversely affects the stress transfer between the fibril in some loading directions, thereby affecting the overall stress development in the fiber under tensile deformation.

The middle lamella joining the ultimate cells is observed to become more plastic as well as homogeneous due to the gradual elimination of micro-voids, while the ultimate cells are only affected slightly.

The increase in percentage crystallinity index through alkali treatment occurs because of the removal of cemented materials, which leads to a better packing of cellulose chains.

Reductions in microfibril angle/spiral angle improvs load alignment by transferring load closer to the fiber axis and therefore increasing molecular orientation.

Again, this allows the crystallites to align more freely under load when not in the composite form. Other treatments with a dilute resin solution are sometimes applied to encompass the crystallites and the extra surface area of the fiber internals to improve interfacial strength.

These imperfections may have been in the fibers previously; however the removal of the surface matter makes them more apparent. Additional imperfections may be the result of handling during processing or over treatment. Can lead to improved 'interlocking' between fiber and the resin when in composite forms.

With the removal of hemi-cellulose, this makes fibers far more stable when exposed to moisture and has been reported by a number of authors.

Over alkalization of cellulosic fibers alters the alpha cellulose to cellulose-II in the fibrils. This is not desired as the alpha cellulose displays greater alignment in crystalline structure, and exhibits more desirable mechanical properties.

his swelling has been reported to be from a reaction in which the natural crystalline structure of the cellulose relaxes. The type of Alkali ( $\mathrm{KOH}, \mathrm{LiOH}, \mathrm{NaOH})$ and its concentration will influence the degree of swelling and hence the degree of lattice transformation to cellulose-II.

It has been suggested that when swollen the fibers should not be put under tension as they are very vulnerable due to transformations occurring in the crystalline structure.

Swelling of the fiber in the composite can cause micro cracking in the matrix. This has been reported to be from poor washing of the fibers after alkalization.

There are various alkali solutions that can be used including hydrogen peroxide $\left(\mathrm{H}_{2} \mathrm{O}_{2}\right)$ and sodium hydroxide $(\mathrm{NaOH})$. Hydrogen peroxide [2] is used much less frequently due to the dangers associated with its use. Sodium hydroxide however is most commonly used for fiber pre-treatment and is freely obtainable in the form of caustic soda.

From Table 7, NaOH has substantial effects on improving mechanical properties, even with low treatment concentrations and process times. With the correct pre-treatment 
process, a $30 \%$ increase in interfacial strength in the composite can be generally achieved, with higher mechanical properties of the fibers also observed. The general concentration range of $\mathrm{NaOH}$ from the articles investigated seems to be $1-25 \%$ and processing time of 1-60 min. It can also be highlighted that minimal effects are reported towards higher process times above $60 \mathrm{~min}$ [18], and even above $30 \mathrm{~min}$ treatment time, little resultant effect has been noticed in flax, even at low alkali concentrations. Figure 10 gives the constituent breakdown of the fibers and aids in understanding of the required alkalization process conditions for each fiber as hemicellulose/cellulose content ratio varies between fibers.

\section{The Alkalization Process}

From the information taken from the literature study, an alkalization process time of 10,20 , and 30 min time in $3 \% \mathrm{NaOH}$ solution was deemed to be a reasonable study to gain feeling for the process. In reality however, due to the differences in constituents between each natural fiber shown in Figure 10, optimal process conditions including treatment

Table 7. Alkalization literature information to aid in compilation of testing conditions.

\begin{tabular}{|c|c|c|c|c|c|}
\hline Fiber & Alkali & Concentration & $\begin{array}{l}\text { Treatment } \\
\text { times }\end{array}$ & Ref. & Comments/Effect \\
\hline Coir & $\begin{array}{l}\text { Sodium hydroxide } \\
(\mathrm{NaOH})\end{array}$ & $5 \%$ Aqueo & $0-200 \mathrm{~h}$ & [18] & $\begin{array}{l}\text { UTS increases from } \\
200-225 \mathrm{MPa} \text { at } 100 \mathrm{~h} \text { then } \\
\text { begins to decrease }\end{array}$ \\
\hline Sisal & $\begin{array}{l}\text { Sodium hydroxide } \\
\qquad(\mathrm{NaOH})\end{array}$ & Aque & $0-120 \mathrm{~h}$ & [18] & $\begin{array}{l}\text { Steady increase tensile strength } \\
\text { from } 500 \mathrm{MPa}-1100 \text { at } 90 \mathrm{~h} \text {. } \\
\text { Decreases after this. }\end{array}$ \\
\hline Sisal & $\begin{array}{l}\text { Sodium hydroxide } \\
\qquad(\mathrm{NaOH})\end{array}$ & w/v & $\begin{array}{c}1 \mathrm{~h} \text { at } 25^{\circ} \mathrm{C}, \\
\text { dried at } 60^{\circ} \mathrm{C} \text { for } \\
24 \mathrm{~h}\end{array}$ & {$[21]$} & $\begin{array}{l}\text { Research into improving bond } \\
\text { strength. } 30 \% \text { increase in } \\
\text { interfacial shear strength. }\end{array}$ \\
\hline $\begin{array}{l}\text { Flax } \\
\text { (loose fiber) }\end{array}$ & $\begin{array}{l}\text { Sodium hydroxide } \\
(\mathrm{NaOH})\end{array}$ & $1,2,3 \%$ & $20 \min$ & [22] & $\begin{array}{l}\text { Washed in acified water } \\
\text { ( } \mathrm{HC} 10.1 \mathrm{M} \text { in } 1 \mathrm{~L} \text { of water). } \\
\text { Dried at } 80^{\circ} \mathrm{C} \text { for } 8 \mathrm{~h} \text {. Increase in } \\
\text { both longitudinal and transverse } \\
\text { strength in composite. }\end{array}$ \\
\hline Flax & $\begin{array}{l}\text { Sodium hydroxide } \\
(\mathrm{NaOH})\end{array}$ & $1,2,3 \%$ & $20 \min$ & {$[23]$} & $\begin{array}{l}\text { Washed in cold water, then } \\
\text { acified water, then washed } \\
\text { again. } 30 \% \text { increase in long- } \\
\text { itudinal strength at } 3 \% \text { solution. }\end{array}$ \\
\hline & $\begin{array}{l}\text { Sodium hydroxide } \\
\qquad(\mathrm{NaOH})\end{array}$ & $10-25 \%$ & $\begin{array}{l}1-30 \text { min with } \\
\text { intermediate } \\
\text { intervals }\end{array}$ & {$[26]$} & $\begin{array}{l}\text { Optimum result obtained at } \\
16 \% \mathrm{NaOH} \text { solution. Too high } \\
\text { concentration leads to degrada- } \\
\text { tion. Treatments longer than } \\
10 \text { min have only minor added } \\
\text { effect }\end{array}$ \\
\hline Flax & $\begin{array}{l}\text { Sodium Hydroxide } \\
\qquad(\mathrm{NaOH})\end{array}$ & $\begin{array}{l}\text { 'Boiled' in } 2- \\
10 \% \text { solution }\end{array}$ & $30 \mathrm{~min}$ & {$[25]$} & $\begin{array}{l}\text { Washed in distilled water. Hurds } \\
\text { removed. Fibers 'purified'; fiber } \\
\text { separation increased. }\end{array}$ \\
\hline $\begin{array}{l}\text { Flax, } \\
\text { hemp, jute, } \\
\text { and sisal }\end{array}$ & $\begin{array}{l}\text { Sodium hydroxide } \\
\qquad(\mathrm{NaOH})\end{array}$ & $\begin{array}{l}\text { Only states } \\
\text { 'mild' }\end{array}$ & $60 \mathrm{~min}$ & {$[24]$} & $\begin{array}{l}\text { Rinsed with distilled water con- } \\
\text { taining a small amount of acetic } \\
\text { acid. Generally } 10 \% \text { increase in } \\
\text { composite properties. }\end{array}$ \\
\hline
\end{tabular}


times and $\mathrm{NaOH}$ concentrations will vary for each fiber. In this case the parameters for the study will remain consistent to allow for comparison of alkalization effect between fibers. The experimental process used is given below in Table 8 and was carried out for flax, abaca, kenaf, and sisal.

After conducting the alkalization of the natural fibers as in Table 8, tensile testing was carried out according to ASTM D 3822-01 using the same procedure as in the previous fiber moisture studies. The results of this testing can be found in the following section.

\section{Alkalization Test Results - Tensile Strain to Failure}

The results of alkalization on the tensile failure strain of the natural fibers flax, abaca, kenaf, and sisal can be viewed in Table 9. These results are additionally shown graphically in Figure 11.

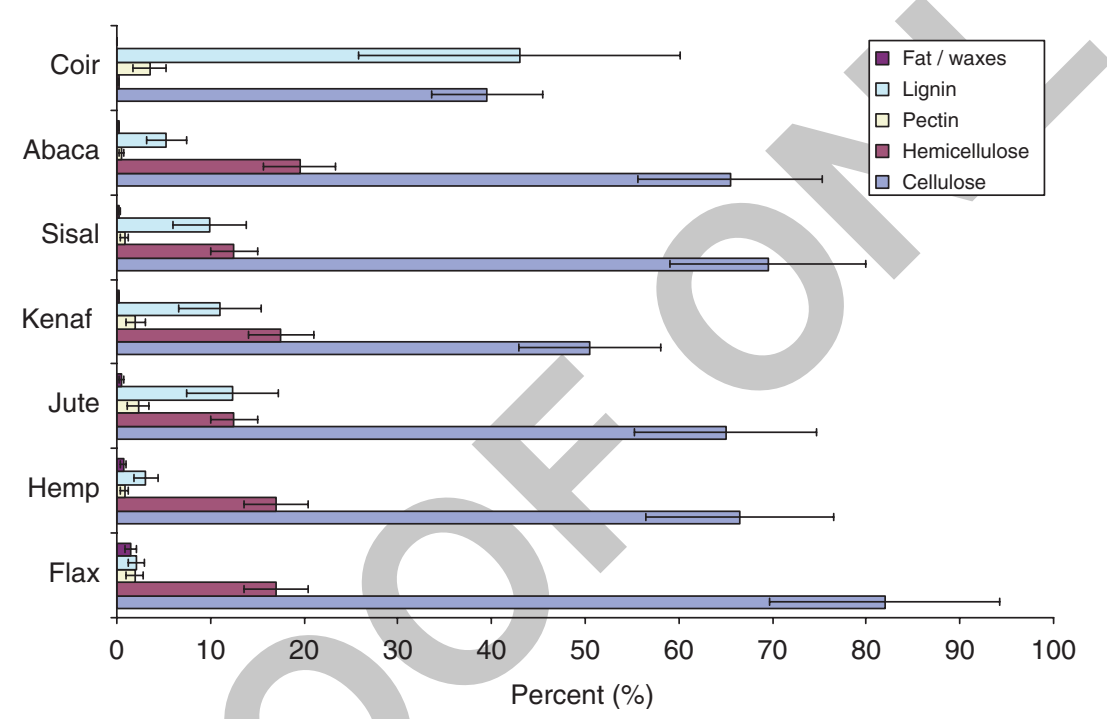

Figure 10. Percentage constituent make-up of the target fibers. Error bars are given to show how properties vary for each of the constituent ratios.

Table 8. The alkalization process steps used.

\begin{tabular}{|c|c|c|}
\hline Step & Action & Comment \\
\hline 1 & Take fibers and dry to consistent moisture content & In oven at $60^{\circ} \mathrm{C}$ for $1 \mathrm{~h}$ \\
\hline 2 & $\begin{array}{l}\text { Take fiber samples and immerse in } 3 \% \mathrm{NaOH} \\
\text { solution }\end{array}$ & 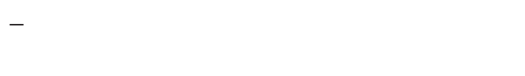 \\
\hline 3 & After 10 min take out sample of each fiber type & $\begin{array}{l}\text { Wash in slightly acidic solution and } \\
\text { cleanse thoroughly with ionized water. }\end{array}$ \\
\hline 4 & After 20 min take out sample of each fiber type & $\begin{array}{l}\text { Wash in slightly acidic solution and } \\
\text { cleanse thoroughly with ionized water. }\end{array}$ \\
\hline 5 & After 30 min take out sample of each fiber type & $\begin{array}{l}\text { Wash in slightly acidic solution and } \\
\text { cleanse thoroughly with ionized water. }\end{array}$ \\
\hline 6 & Leave fibers to dry naturally & - \\
\hline 7 & Dry fibers to consistent moisture level & In oven at $60^{\circ} \mathrm{C}$ for $1 \mathrm{~h}$ \\
\hline 8 & Organize and test fibers as before & Using ASTM D 3822-01 \\
\hline
\end{tabular}


Table 9. Tensile strain to failure results for $\mathrm{NaOH}$ treated fiber including standard deviation.

\begin{tabular}{lllll}
\hline Fiber & Untreated & \multicolumn{1}{c}{ 10 $\mathbf{~ i n}$} & \multicolumn{1}{c}{ 20 $\mathbf{~ m i n}$} & \multicolumn{1}{c}{ 30 $\mathbf{~ i n}$} \\
\hline Flax & $2.54 \pm 1.112$ & $1.95 \pm 0.632$ & $1.92 \pm 0.587$ & $1.61 \pm 0.489$ \\
Abaca & $4.95 \pm 1.65$ & $3.14 \pm 0.794$ & $3.17 \pm 0.578$ & $2.39 \pm 0.768$ \\
Kenaf & $1.98 \pm 0.427$ & $1.06 \pm 0.423$ & $1.37 \pm 0.216$ & $1.37 \pm 0.33$ \\
Sisal & $2.82 \pm 1.182$ & $2.13 \pm 0.502$ & $1.86 \pm 0.32$ & $1.6 \pm 0.366$ \\
\hline
\end{tabular}

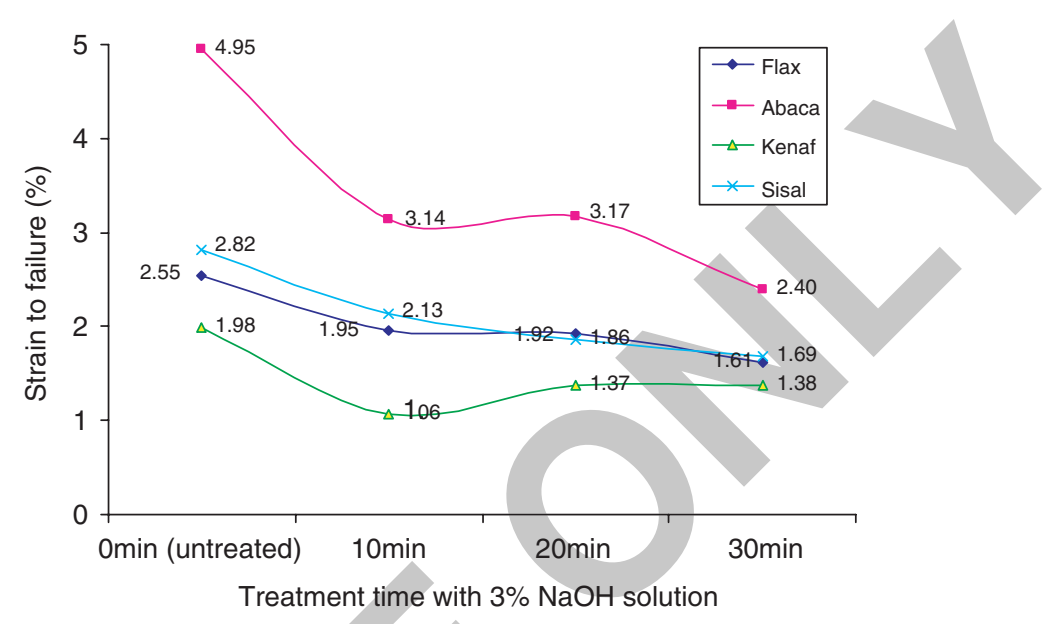

Figure 11. The effect of $3 \% \mathrm{NaOH}$ treatment on tensile strain to failure of chosen natural fibers.

From initial observation, it is apparent that as treatment time is increased, the fiber strain at maximum load decreases and in the case of abaca, reduced by nearly $50 \%$ in comparison to the untreated fiber. In the case of sisal and flax, there seems to be a steadier decrease in strain at max load according to treatment time with $\mathrm{NaOH}$ solution. The decrease in ability for the natural fibers to deform and strain may be attributed to the removal of the hemicelluloses during processing which would normally bind between the microfibrils and provide a supportive matrix to allow the fibers to deform to a greater extent during loading. In Figure 12 it is interesting to observe the changes in standard deviation between the treatment times. In general it is observed that after alkalization treatment the standard deviation for the fibers strains at failure all reduce to a reasonable extent. This indicates that through the removal of hemicelluloses, fats and waxes through alkalization, the predictability margin of failure for the base fibers are narrowing. In the case of sisal it has reduced remarkably with the standard deviation falling in the region of $70 \%$ after 30 min of treatment.

\section{Alkalization Test Results - Tensile Strength}

The results of alkalization on the fiber tensile strength of the natural fibers flax, abaca, kenaf, and sisal can be viewed in Table 10. These results are additionally shown graphically in Figure 13. From analzsing the results, it can be seen that the flax and abaca generally seem to lose fiber strength as the alkalization time increases. In the case of kenaf, 
Tensile Testing of Cellulose Based Natural Fibers

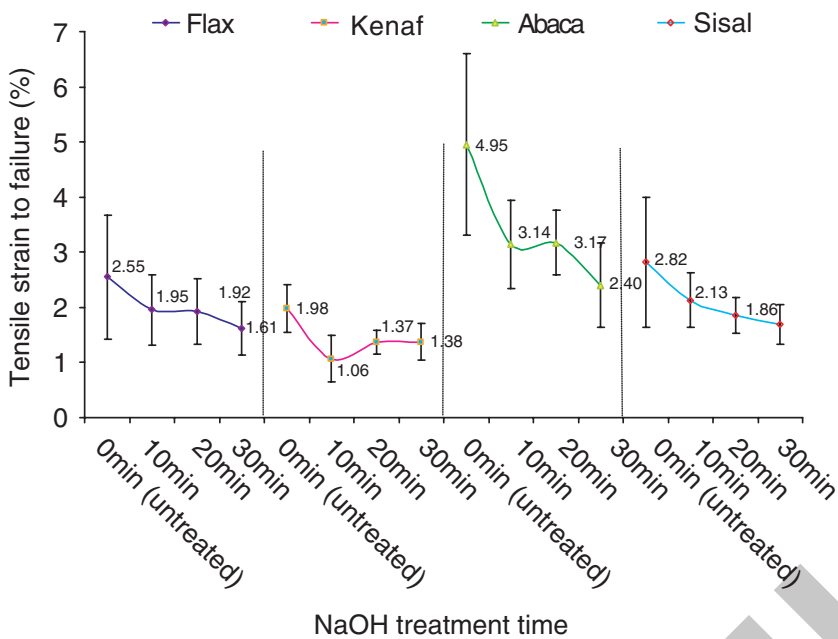

Figure 12. The effect of $3 \% \mathrm{NaOH}$ treatment on the tensile strain to failure showing the standard deviation.

Table 10. Tensile strength results table for $\mathrm{NaOH}$ treated fiber including standard deviation.

\begin{tabular}{lcrrr}
\hline Fiber & Untreated & \multicolumn{1}{c}{ 10 min } & \multicolumn{1}{c}{ 20 min } & \multicolumn{1}{c}{ 30 min } \\
\hline Flax & $1632.24 \pm 937$ & $1188.95 \pm 461$ & $1112.77 \pm 702$ & $722.74 \pm 455$ \\
Abaca & $945.70 \pm 299.7$ & $778.48 \pm 242$ & $513.50 \pm 241$ & $549.5 \pm 331$ \\
Kenaf & $473.27 \pm 241$ & $481.6 \pm 133$ & $417.4 \pm 152$ & $419.72 \pm 240$ \\
Sisal & $546.31 \pm 318.55$ & $776.13 \pm 303$ & $500.72 \pm 147$ & $502.72 \pm 331$ \\
\hline
\end{tabular}

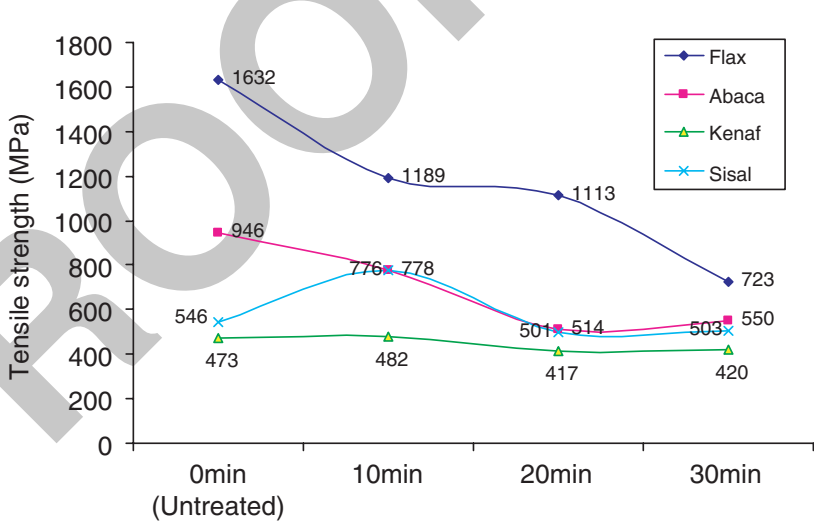

Treatment time with $3 \% \mathrm{NaOH}$ solution

Figure 13. The effect of $3 \% \mathrm{NaOH}$ treatment on tensile strength of chosen natural fibers.

maximum stress seems to remain similar for all treatment times. However it is interesting to see that sisal increases in failure strength after $10 \mathrm{~min}$, however dips back to similar values to the untreated base fiber for 20 and 30 min alkalization duration.

As the fibers are tested at similar moisture contents due to oven drying before testing, this effect is therefore unlikely to be caused by swelling of the fiber from moisture differences. 
In Figure 14 below, the upturn in the sisal fiber can also be observed to have the narrowest strength deviation band of the four points. It is also interesting to note a general pattern from the standard deviations for the four fibers. It seems, in general, that alkalization reduces the deviation of failure stress from the untreated base fibers through all the treatment durations. However, it is also noticed for kenaf, abaca, and sisal that the deviation increases after 30 min treatment with $\mathrm{NaOH}$, which may imply damage to the structure of the fibers in some way.

\section{Alkalization Test Results - Young's Modulus}

The results of alkalization on the Young's modulus of the natural fibers flax, abaca, kenaf, and sisal can be viewed in Table 11 . These results are additionally shown graphically in Figure 15. After $10 \mathrm{~min}$ of treatment time both kenaf and sisal increase significantly in modulus. for the natural fibers flax and abaca, young's modulus remains similar to the untreated fibers for $10 \mathrm{~min}$ treatment time, with the modulus dipping for the $20 \mathrm{~min}$ alkalization period. After $30 \mathrm{~min}$, the modulus of Flax continues to drop, where conversely that of kenaf seems to rise to approximately a similar modulus of the untreated fiber.

Interestingly, when considering Table 6 and the observed effects from alkalization in literature, some speculations of cause of modulus increase can be made. Observations (1),

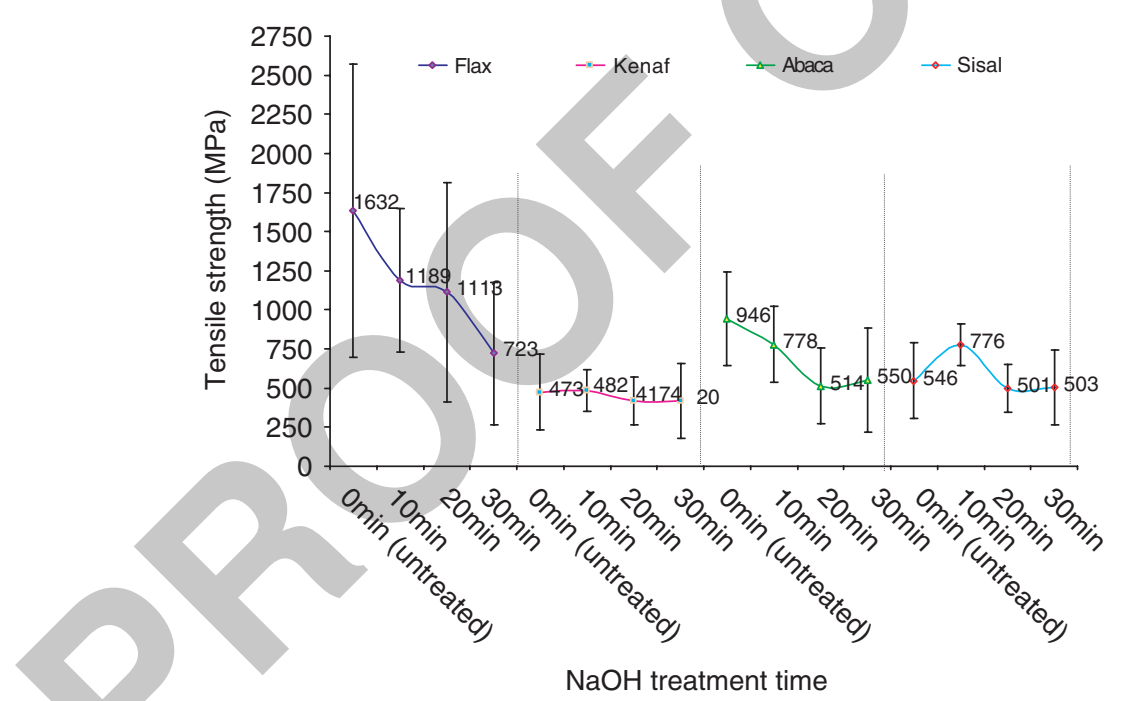

Figure 14. The effect of $3 \% \mathrm{NaOH}$ treatment on the tensile strength showing standard deviation.

Table 11. Young's modulus results for tensile testing of $\mathrm{NaOH}$ treated fiber including standard deviation.

\begin{tabular}{lcccc}
\hline Fiber & Untreated & \multicolumn{1}{c}{ 10 $\mathbf{~ m i n}$} & \multicolumn{1}{c}{ 20 $\mathbf{~ m i n}$} & \multicolumn{1}{c}{ 30 $\mathbf{~ i n}$} \\
\hline Flax & $66.19 \pm 22.23$ & $68.15 \pm 17.47$ & $64.32 \pm 44.2$ & $51.51 \pm 24.9$ \\
Abaca & $23.43 \pm 6.466$ & $25.63 \pm 11.32$ & $18.14 \pm 8.3$ & $25.48 \pm 12.2$ \\
Kenaf & $34.21 \pm 17.17$ & $49.49 \pm 17.75$ & $37.27 \pm 15.79$ & $35.04 \pm 14.25$ \\
Sisal & $17.37 \pm 8.415$ & $38.14 \pm 14$ & $28 \pm 6.8$ & $33.17 \pm 12.19$ \\
\hline
\end{tabular}


(2) and (5) all indicate that the removal of the binding hemicelluloses in the interfibrillar regions allow fiber microfibrils to re-arrange themselves and transfer loads more effectively. Consequently this means less collective fiber deformation during loading, allowing microfibrils to perform more efficiently.

From Figure 16 it is apparent that a trend in the standard deviation of the tested fibers is not so obvious. In general, the standard deviations appear reasonably varied for each fiber during treatment time, However there is not a massive difference between each point. In the case of flax however, at 20-minute treatment time, the standard deviation increases dramatically, whilst there is a downturn in average modulus value. This indicates something is changing in the fiber at this point, and it is interesting to note from Table 6 in point (9) that alkalization of natural fibers can alter the alpha cellulose to cellulose-II in the fibrils. Alpha cellulose in general has more desirable mechanical properties than

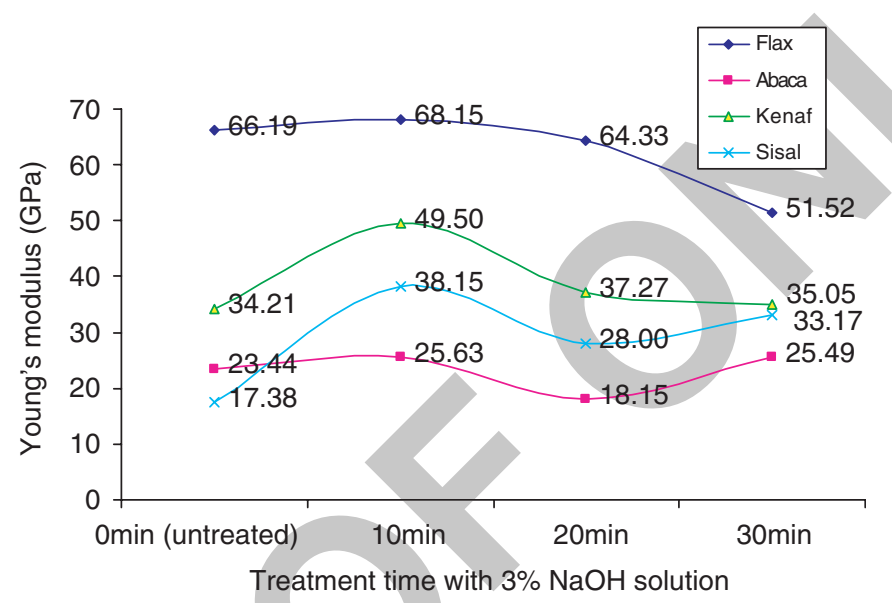

Figure 15. The effect of $3 \% \mathrm{NaOH}$ treatment on Young's modulus of chosen natural fibers.

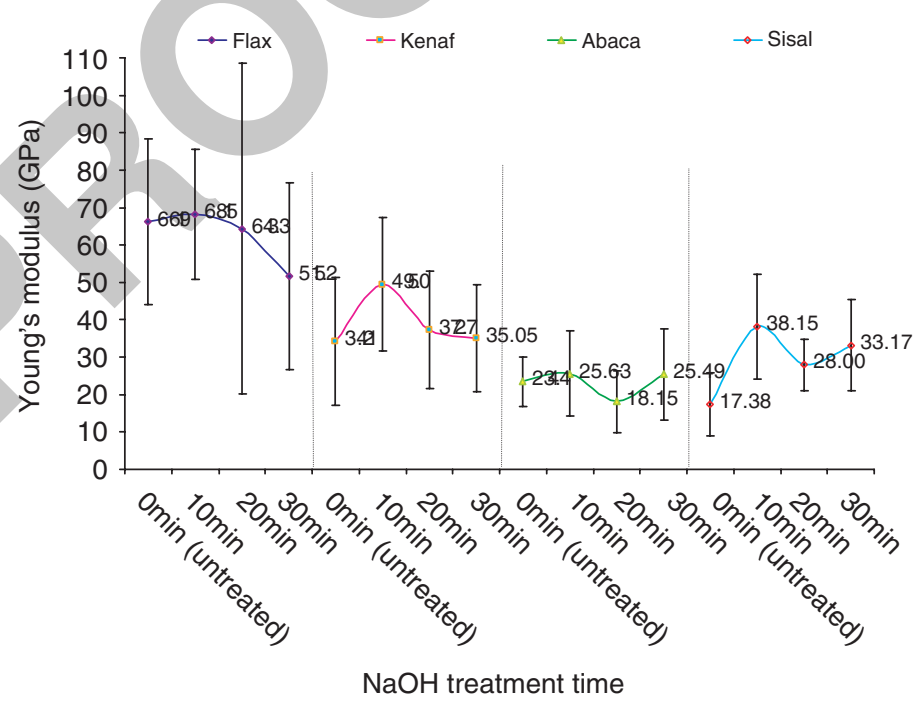

Figure 16. The effect of $3 \% \mathrm{NaOH}$ treatment on the Young's modulus showing standard deviation. 
cellulose-II, and the large increase in standard deviation at this point may mark a transition point of the cellulose type at this treatment time, and hence a variability in failure stress and Young's modulus.

During all the testing of the natural fiber, through moisture studies and also alkalization, there has been an endeavour to reduce any source of error in the results. From comparison of the testing results to those in the literature, most of the tensile testing results are deemed to be reasonable. However, for completeness a list of sources of inaccuracy, with some sources being unavoidable, are given in the following section.

\section{DISCUSSION}

There are a number of possible inaccuracies that could be argued that affect the overall accuracy or validity of results. These are mostly associated with the technical difficulties due to the nature of natural fibers. It is felt these problems are best outlined for discussion and debate; therefore a round up of the sources of error and inaccuracies is given in Table 12 .

It is hoped from outlining the possible sources of error the problems associated with the testing of natural fibers is better understood and therefore better able to be overcome. It is however thought that the results obtained from the testing are of reasonable enough accuracy to confirm that they fall within the spread of those observed in the academic community.

From the tensile testing carried out, the values for the mechanical properties of natural fibers under various conditions have been obtained. This is of prime importance as these values and understanding provides the basis of all the work to be carried out. Furthermore, an understanding that moisture has a distinct effect on natural fiber is gained. Although these patterns are not entirely clear or conclusive, it provides some insight and speculation into the important issue of understanding the structural behavior of the fibers under various environmental conditions. It is also apparent that the process of fiber alkalization is complex, and from the literature study it is apparent if it is not carried out with care as to process time and alkali concentration, it is easy to induce undesired effects on the fibers.

The fibers tested, in general exhibit qualities that perhaps make them suitable for certain composite forms and applications. Jute seems to be a favourite fiber for development in the academic community at present, and it is thought that interest is circled around jute due to the apparent stability in mechanical strength to moisture exposure, as well as low cost. Kenaf is also being recognized for these applications, and whilst the mechanical properties are not as good as some of the other target fibers, the reliability seems to be valued considerably by developers.

From the study on mechanical properties it is also interesting to evaluate the energy until failure and this can be observed in Figure 17. The fiber that stands out from the rest in terms of performance is abaca. However the relatively low Young's modulus may make it unsuitable for some composite applications. Flax again has proved to be one of the more exciting fibers tested in this study; however it is also of higher cost than the other fibers, but the potential is great.

From working and handling flax during testing, its inherent strength is immediately apparent. Some of the values returned during the testing, although sparse and scattered, were exceptional. This is giving glimpses into the fiber's potential, and hints that if 
Table 12. Possible sources of inaccuracy in the work undertaken for tensile testing.

Problem
description Inaccuracy effect

Cross-sectional Due to the nature of natural fibers being made up of smaller networks of fibers, the area measurement actual CSA of the fiber will not be a uniform area, making it hard to predict with exact accuracy. Additionally, the measuring of natural fibers with callipers proves a skill, and any mistakes may result in values being different. The CSA of the general fibers is also not always circular, therefore it has to be said that any measurement of area is an educated estimate and not exact.

Cross-sectional Due to the fiber being under load at failure point, it may be the case that there has been area at failure point an area reduction from the original measurement due to sub failures of microfibrils in the fiber. Thus the cross-sectional area at failure may not be the same as that before loading. If the CSA is altered so is the stress at failure, leading to an error in results.

What constitutes When working with the natural fibers it becomes apparent very quickly that it is not a fiber? always clear as to what constitutes a fiber. For example if a flax fiber is pulled apart, a netted structure of sub structures within the fiber is found. These are made up of the long microfibril cells. So this raises the question, what constitutes a fiber, and what does the academic community constitute as a fiber? What parts of the fiber are being tested to obtain their mechanical properties to compare values with? Unless this is exactly known, like with like may not be compared.

Humidity measurement/ variability

Errors in the estimation of humidity could occur, affecting the results of the humidity comparisons for the mechanical properties of the fibers. This may be due to reading equipment, the influence of desiccators shape/contents on the exposure level. The humidity estimation for humidity exposure at room conditions in the university may not be completely accurate as it is variable from day to day, and therefore the value below $65 \%$ humidity may be too low. It is also open to debate how variable room humidity will translate to an equivalent humidity exposure on the fibers.

Natural Natural fibers' properties vary a reasonable amount from fiber to fiber. For example, fiber variability within a batch of Jute fiber, small clumps of fibers may have different qualities from other clumps although possibly taken from the same plant. When testing the fibers and comparing humidity properties weaker or stronger clumps within the same batch may be tested. It is hoped that testing a number of random fibers within the batch to increase the spread has helped to combat this. Nonetheless this has to be highlighted as a possible source of error.

Damage

Damage from handling during testing is kept to a minimum. However, fibers may be

during handling pre-damaged from mishandling by others in the supply chain. This may not be a terrible thing as in reality rough handling will be apparent in most manufacturing process that the fiber is part of and returned mechanical properties will reflect the fiber's ability to deal with this mishandling.

Temperature in oven

To maintain given humidity in the desiccators, different temperatures had to be used for $90 \%$ and $65 \%$ test specimens in conjunction with a specific chemical solution to provide the desired humidity. It is not totally understood if the differences in temperature as well as humidity would have an effect on the mechanical properties of the fiber and should be considered when looking at any unusual results.

Dessicator

It is not fully understood if the dessicator solutions used to create the desired humidity in solution the oven would affect the fiber properties, and therefore the validity of the testing results. Reassurances were however given by the technician involved creating the solutions.

knowledge can be carefully applied, the potential of the fiber can be reached to create a value added product suitable for structural applications. This is expected to be especially so when combining nanotechnology to combat the weaknesses of natural fiber composites which include poor impact strength and low fire resistance. In future work in composite manufacture it will be extremely interesting to see how each of these fibers perform in 


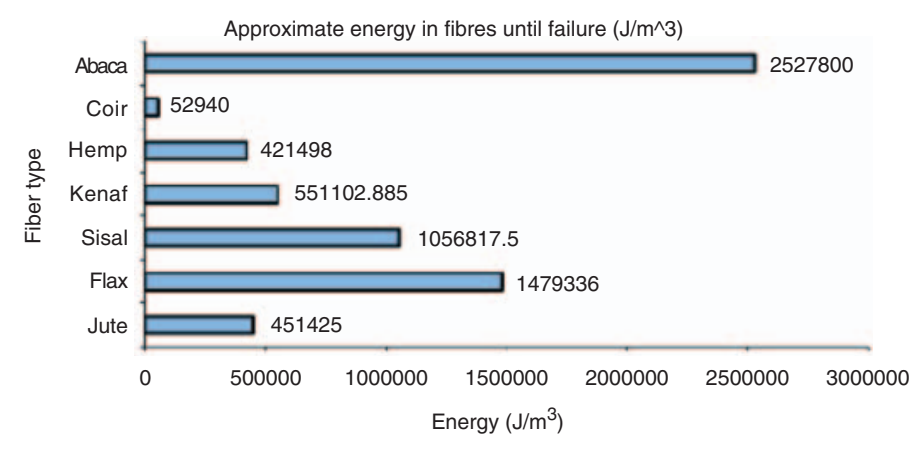

Figure 17. Approximate energy stored until failure of each base natural fiber (untreated).

composite form, and it if they still hold their order of mechanical properties in composite form between fiber types. It may also be the case that some of the exciting properties of the base fiber are initially lost in composite form, and the challenge will be to apply pre-processing knowledge and research to unlock their full potential.

\section{CONCLUSIONS}

From the tensile testing carried out project values for the mechanical properties have been obtained for natural fibers under various conditions. This is of prime importance as these values and understanding provide the basis of all future work to be carried out on the fibers, and how these properties may affect the performance of the composite material. Furthermore, an understanding of how moisture affects some of the fibers is gained. Although these moisture patterns are not entirely clear or conclusive, it provides some insight and speculation into the important issue of understanding the structural behavior of the fibers under various environmental conditions. It can also be concluded that the process of fiber alkalization has an effect on the base strength of natural fiber, and there may be an optimum process condition if performance of the fiber in composite material form is to be maximized.

It is also apparent that there are many potential sources of error in the results, and that are associated with the difficulty in testing natural fibers in particular. These have been outlined for discussion, and will hopefully aid the academic community in overcoming these problems. However, the work carried out should give a good basis for comparison between sourced fibers. Additional reassurance is given in that the mechanical properties available in the literature generally cohere to tested values. It is hoped the results of this article may allow work to move further towards developing exciting natural fiber composites enhanced with nanotechnolgy.

\section{ACKNOWLEDGMENTS}

The authors would like to thank the Engineering and Physical Sciences Research Council (EPSRC) for funding the research as well as Wigglesworth Ltd and Toyota Auto Body (Japan) for aiding in fiber supply. 


\section{REFERENCES}

1. Murali Mohan Rao, K. and Mohana Rao, K. (1984). Extraction of The Tensile Properties of Natural Fibers, Vakka, Date and Bambo, Journal of Composite Structures, 77(2007): 288-295.

2. Various Contributors, In: Franck, Robert R. (ed.), Bast and Other Plant Fibers, Textile Industry Publication, CRC \& Woodhead Publishing Limited, ISBN 1855736845.

3. Various Contributors, In: Anthony Kelly (ed.), The Concise Encyclopedia of Composite Materials, Revised edn, Pergamon Publications, ISBN 0080423000.

4. Joseph, S., Sreekala, Oommen, Z., Kosy, P. and Thomas S. (2002). A Comparison of Mechanical Properties of Phenol Formaldehyde Composites Reinforced with Banana Fibers and Glass Fibers, Composites Science and Technology, 62(14): 1857-1868.

5. Pothan L.A., Oonmmen, Z. and Thomas, S. (2003). Dynamic Mechanical Analysis of banana Fiber Reinforced Polyester Composites, Composites Science and Technology, 63(2): 283-293.

6. Pothan, L.A. and Thomas, S. (2003). Polarity Parameters and Dynamic Mechanical Behavior of Chemically Modified Banana Fiber Reinforced Polyester Composites, Composites Science and Technology, 63(9): 1231-1240(10).

7. Geethamma, V.G. and Mathew, K.T. (1998). Composite of Short Coir Fibers and Natural Rubber - Effect of chemical Modification, Loading and Orientation of Fiber, Polymer, 39(6): 1483-1491(9).

8. Savastano, H., Agopyan, Nolasco, A.M. and Pimentel, L. (1999). Plant Fiber Reinforced Cement Components for Roofing, Construction and Building Materials, 13(8): 433-438(6).

9. Savastano, H., Agopyan. (1999). Transition Zone Studies of Vegetable Fiber-cement Paste Composites, Cement and Concrete Composites, 21(1): 48-57(9).

10. Baley, C. (2002). Analysis of the Flax Fibers Tensile Behavior and Analysis of the Tensile Stiffness Increase, Composites Part A, 33(7): 939-948(10).

11. Eichhorn, S.J. and Young R.J. (2004). Composite Micromechanics of Hemp Fibers and Epoxy Resin Microdroplets, 64(5): 767.

12. Keller, A. (2003). Compounding and Mechanical Properties of Biodegradable Hemp Fiber Composites, Composite Science and Technology, 63(9): 1307-1316(10).

13. Li, Z., Wang, X. and Wang, L. Properties of Hemp Fiber Reinforced Concrete Composites, Fibers and Polymers, 5(3): 187-197.

14. Kumar, A.P., Singh, R.P., Sarwade, Bhimrao, D. (2005). Degradability of Composites, Prepared from Ethylene-Propylene Copolymer and Jute Fiber Under Accelerated Aging and Biotic Environments, Materials Chemistry and Physics, 92(2-3): 458-469.

15. Gassan, J. and Bledzki, A.K. (1999). Possibilities for Improving the Mechanical Properties of Jute-epoxy Composites by Alkali Treatment of Fibers, Composites Sciences and Technology, 59(9): 1303-1309(7)

16. Eichhorn, S.J., Baillie, C.A., Mwaikambo, L.Y., Ansell, M.P. and Dufrese, A. (2001). Current International Research into Cellulostic Fibers and Composites, Journals of Materials Science, 36: $2107-2131$.

17. Olesen, P.O. and Plackett, D.V. (1999). Perspectives on the Performance of Natural Plant Fibers Plant Fiber Laboratory, Royal Veterinary and Agricultural University, Copenhagen, Denmark, Essentials for the Future, 27-28 May.

18. Bledzki, A.K. and Gassan, J. (1999). Composites Reinforced with Cellulose Based Fibers, Prog. Polymer Science, 24: 221-274.

19. Roberts Joffe, Janis Andersons and Lennart Wallstrom (2003). Strength and Adhesion characteristics of Elementary Flax Fibers with Different Surface Treatments, Composites: Part A, 34: 603-612.

20. Eichhorn, S.J., Baillie, C.A., Mwaikambo, L.Y., Ansell, M.P. and Dufrese, A. (2001). Current International Research into Cellulostic Fibers and Composites, Journals of Materials Science, 36: 2107-2131. 
21. Valadez-Gonzalez, A., Cervantes-Uc, J.M., Olayo, R. and Herrara-Franco, P.J. (1999). Effect of Surface Treatment on Fiber - Matrix Bond Strength of Natural Fiber Reinforced Composites, Composites: Part B, 30: 309-320.

22. Vande Weyenberg, I., Chi Truong, T., Vangrimde, B. and Verpoest, I. (2006). Improving the Properties of UD Flax Fiber Reinforced Composites by Applying and Alkaline Fiber Treatment, Composites: Part A, 37: 1368-1376.

23. Vande Weyenberg, I., Ivens, J., de Coster, A., Kino, B., Baetens, E. and Verpoest, I. (2003). Influence of Processing and Chemical Treatment of Flax fibers on their Composites, Composites Science and Technology, 63: 1241-1246.

24. Panigrahi, S., Powell, T., Wang, B., Tabil, L.G., Crerar, W.J. and Sokansanj, S. The Effects of Chemical Pre-treatment on Flax Fiber Bio-composites, ASAE Meeting Presentation, Paper Number - RRV03-0018.

25. Alexander Bismark, Ibon Aranberri-Askargorta and Jurgen Spinger (2002). Surface Characterisation of Flax, Hemp and Cellulose Fibers: Properties and Water Uptake Behavior, Polymer Composites, 23(5).

26. Borysiak, S. and Garbarczyk, J. (2003). Applying the WAXS Method to Estimate the Supermolecular Structure of Cellulose after Mercerisation, Fibers and Textiles Eastern Europe, 11(5): 104-106. 